\title{
Pollutant's Horizontal Dispersion Along and Against Sinusoidally Varying Velocity from a Pulse Type Point Source
}

\author{
Mritunjay K. SINGH ${ }^{1}$, Nav K. MAHATO ${ }^{2}$, and Naveen KUMAR ${ }^{3}$ \\ ${ }^{1}$ Department of Applied Mathematics, Indian School of Mines, Dhanbad, India \\ e-mail: drmks29@rediffmail.com (corresponding author) \\ ${ }^{2}$ Department of Mathematics, C.V. Raman College of Engineering, \\ Bhubaneswar, India; e-mail: kr.nav81@gmail.com \\ ${ }^{3}$ Department of Mathematics, Banaras Hindu University, Varanasi, India \\ e-mail:nks_1953@yahoo.co.in
}

\begin{abstract}
An analytical solution of a two-dimensional advection diffusion equation with time dependent coefficients is obtained by using Laplace Integral Transformation Technique. The horizontal medium of solute transport is considered of semi-infinite extent along both the longitudinal and lateral directions. The input concentration is assumed at an intermediate position of the domain. It helps to evaluate concentration level along the flow as well as against the flow through one model only. The source of the input concentration is considered to be of pulse type. In the presence of the source, it is assumed to be decreasing very slowly with time, and just after the elimination of the source it is assumed to be zero. The dispersion coefficient and the advection parameter are considered directly proportional to each other. The analytical solution may be used to predict the solute concentration level with position and time in an open medium as well as in a porous medium. The effect of heterogeneity on the solute transport may also be predicted.
\end{abstract}

Keywords: solute transport, aquifer, input concentration, heterogeneity, Cartesian system.

Ownership: Institute of Geophysics, Polish Academy of Sciences;

(C) 2015 Singh et al. This is an open access article distributed under the Creative Commons Attribution-NonCommercial-NoDerivs license,

http://creativecommons.org/licenses/by-nc-nd/3.0/. 


\section{INTRODUCTION}

Pollutant mass originating from a variety of natural and anthropogenic sources (volcano, industries, factories, refineries, sewage systems, garbage disposal sites, mines, etc.) is a major cause of degradation of the environment of air, surface water, soil, and groundwater. Mathematical modelers use the advection diffusion equation (ADE) to describe the concentration level of the pollutant mass at different position and time, away from its source, through its analytical and numerical solutions. In one-dimensional Cartesian system of coordinates, $\mathrm{ADE}$ is as follows

$$
\frac{\partial c}{\partial t}=\frac{\partial}{\partial x}\left(D \frac{\partial c}{\partial x}-u c\right)
$$

where $c\left[M L^{-3}\right]$ denotes the solute concentration in the medium at any time $t[T]$, and at a position $x[L] ; D\left[L^{2} T^{-1}\right]$ and $u\left[L T^{-1}\right]$ represent the dispersion coefficient and the advection parameter, respectively, along the longitudinal direction ( $x$ axis) of the medium. There are basic theories which relate the dispersion coefficient $D$ and velocity $u$ occurring as the two coefficients in the one-dimensional ADE (Eq. 1): (i) Ebach and White (1958) and Bear (1972) in their one-dimensional analysis suggested that $D$ is proportional to $u$, and (ii) Taylor (1953) in his one-dimensional analysis obtained $D$ proportional to $u^{2}$. Further on, Scheidegger (1957) summarized his analysis on the two possible relationships between $D$ and $u$ according to the role played by molecular diffusion: (i) $D \sim \alpha u^{2}$, where $\alpha$, a constant of the porous medium alone (dynamic dispersivity), is derived by a dynamic procedure applicable when there is enough time in each flow channel for appreciable mixing to take place by molecular transverse diffusion; and (ii) $D \sim \beta u^{2}$, where $\beta$, another constant of the porous medium (geometric dispersivity), is derived by a geometric procedure applicable where there is no appreciable molecular transverse diffusion from one streamline into another. Later on, Freeze and Cherry (1979) have modified these dispersion theories by showing that if the dispersion parameter is proportional to the $n$-th power of the velocity, then $n$ ranges between 1 and 2 .

Dispersion in porous media was significantly discussed by Fried and Combarnous (1971) and groundwater pollution was explored by Fried (1975). The occurrence of anomalous diffusion behavior of an upscaled dispersion coefficient, for a transport process driven locally by a normal diffusion was explained by Matheron and De Marsily (1980). Solute transport in heterogeneous porous formations was discussed by Dagan (1984). An anomalous diffusion model of the local dispersion was also considered for porous media with special properties (Suciu 2014). The apparent behavior of the 
dispersion at larger scales was discussed by Fried (1975), Matheron and De Marsily (1980), Dagan (1984), Suciu (2010, 2014).

The source of advective-diffusive solute mass transport may be a point source, e.g., garbage disposal sites, mines, etc., a line source, e.g., interface of sea water in aquifer or a surface source, e.g., along agriculture field with high doses of chemical fertilizers (Marshal et al. 1996). A point source may be of continuous type or pulse type. In either case, the point source may be uniform or of varying nature. In the latter type, the input concentration may be uniform or of increasing nature in the presence of its source. As soon as the source of pollution is eliminated, the input concentration becomes zero or starts decreasing. Solution of an ADE for a pulse type point source is useful for predicting the rehabilitation time period of a polluted domain once its source is eliminated. Smokes coming out of a chimney, wastes from a drainage system reaching a particular location in rivers, lakes, are examples of uniform pulse-type point sources. As soon as the source is eliminated, the input becomes zero. Infiltration from the ground surface point sources (garbage disposal sites), reaches groundwater level or oil reservoirs, degrading their quality (an example of varying pulse-type point source), where as soon as the source is eliminated, the input, starts decreasing, instead of becoming zero. Only in particular cases it is possible to solve ADE analytically. In a more general situation, numerical techniques are required. The literature presenting most of the analytical methods has been reviewed in a recent work by Guerrero et al. (2009). Many analytical solutions of Eq. 1 in ideal conditions (the two coefficients are independent of the position and time) with growth and decay terms, subject to various initial and boundary conditions in semi-infinite or finite media have been complied (van Genuchten and Alves 1982, Javandel et al. 1984, Domenico and Schwartz 1997). Predicting the fate of pollutants in natural environments, such as rivers and man-made channels, is one of the major concerns. Approximate solutions first began to appear (Banks and Jerasate 1962, Warrick et al. 1971), in which a time-dependent dispersion coefficient was used. Usually, the solute transport models assume a constant dispersion coefficient that is calibrated separately for each different downstream sample location, resulting in different dispersion coefficients for the same flow problem. In an attempt to overcome this, the dispersion coefficient was considered as a function of the mean travel distance successfully (Pickens and Grisak 1981). Another approach is to model dispersivity as a time-dependent function. Based on the observation of numerical results (Suresh Kumar et al. 2008), dispersivity has been suggested to have a time-dependent behavior which reaches asymptotic values after a long time. Additionally, worth mentioning are some of the works solving ADE with variable coefficients analytically in one-dimension (Sander and Braddock 2005, Singh et al. 2008, Chen and Liu 2011, Kumar 
et al. 2011, Chen et al. 2012a, b) and in two- and three-dimensions (Wilson and Miller 1978, Carnahan and Remer 1984, Goltz and Roberts 1986, Yates 1988, Batu 1989, 1993; Leij et al. 1991, Serrano 1995, Aral and Liao 1996, Zou et al. 1996, Zoppou and Knight 1997, Tartakovasky 2000, Singh et al. 2010, Jaiswal et al. 2011, Sudicky et al. 2013).

In the tropical regions, like Indian sub-continent, the water table in the rivers and in the aquifers and its flow is the minimum before the rainy season and is the maximum after the rainy season. But the pollution reaching the river water table through the waste water drainage system or reaching aquifer through infiltration from garbage disposal sites, mines, remain uniform throughout the year. The dispersion of pollutants down the stream and against the stream have a lateral component too, though much weaker in comparison of the longitudinal part. The present study formulates this scenario through a two-dimensional ADE, an initial condition, and boundary conditions, one of which being a pulse type input concentration (the concentration of pollutant at the meeting point in the water table). It is solved using Laplace integral transformation technique. A sinusoidal form of temporally dependent velocity is considered. It represents the minimum and maximum velocity in aquifers as well as in river beds, during the months of summer season (June) and peak of the winter season (December), respectively, in successive years. Of the three dispersion theories discussed in the above paragraph, the dispersion coefficient is considered to be directly proportional to the velocity along both directions. It makes the coefficients of the ADE time-dependent. Such dependence may be used to describe the heterogeneity of the medium and its effect on the solute dispersion. It is valid in porous domain like aquifer and on a surface water body like rivers and lakes. The longitudinal and lateral directions are considered to extend up to infinity. In the presence of the source, the input concentration (due to the source) is considered time-dependent of decreasing nature. The source of pollution is meeting the water medium at a point. To study its concentration distribution behavior along the flow (that is, on the right side domain of the point source), and against the flow (that is, in the domain on the left side), its location is not considered at the origin of the medium as taken in most of the works. It is assumed at an intermediate position of the medium nearer to the origin. In this way, concentration distribution in both situations is studied through one model only. The time-dependent coefficients of the ADE are reduced into constant coefficients by modulating the time variable which takes care of the time-dependent sinusoidal expression. Further on, the two space variables are unified into one space variable and it reduces the twodimensional ADE into one-dimension. 


\section{MATHEMATICAL FORMULATION AND ANALYTICAL SOLUTION}

The two-dimensional ADE with time dependent coefficients may be written as follows:

$$
\frac{\partial c}{\partial t}=D_{x}(t) \frac{\partial^{2} c}{\partial x^{2}}+D_{y}(t) \frac{\partial^{2} c}{\partial y^{2}}-u(t) \frac{\partial c}{\partial x}-v(t) \frac{\partial c}{\partial y} .
$$

The coefficients of second order derivatives are diffusion coefficients and those of first order space derivatives are velocity components along longitudinal and lateral directions, respectively. Let $u$ and $v$ be expressed as

$$
u=u_{0} V(m t) \quad \text { and } \quad v=v_{0} V(m t),
$$

where $m\left[T^{-1}\right]$ may be referred to as unsteady parameter, and $u_{0}$ and $v_{0}$ may be referred to as initial values of $u$ and $v$, respectively. We consider the sinusoidal form of velocity

$$
V(m t)=1-\sin m t .
$$

The different time-dependent forms of velocity expression may also be considered similar to Aral and Liao (1996), based on the properties of algebraic sigmoid function which include the error function. It starts a progress from small beginning, accelerates in the rainy season and reaches up to a limit over a period of time. As the dispersion coefficient is considered directly proportional to velocity, we write

$$
D_{x}=D_{x_{0}} V(m t) \text { and } D_{y}=D_{y_{0}} V(m t),
$$

where $D_{x_{0}}=a u_{0}$ and $D_{y_{0}}=a v_{0}$ are the initial values of $D_{x}$ and $D_{y}$, respectively. Here, $a$ is the dispersivity $[L]$ that depends upon the pore size and geometry. As a result, Eq. 2 may be written as

$$
\frac{1}{V(m t)} \frac{\partial c}{\partial t}=D_{x_{0}} \frac{\partial^{2} c}{\partial x^{2}}+D_{y_{0}} \frac{\partial^{2} c}{\partial y^{2}}-u_{0} \frac{\partial c}{\partial x}-v_{0} \frac{\partial c}{\partial y} .
$$

It is assumed that before the introduction of input concentration, the domain is not solute free. It is uniformly polluted though its level is considered very low. The initial condition is

$$
c(x, y, t)=c_{i} ; \quad 0 \leq x<\infty, 0 \leq y<\infty, t=0 .
$$

The pulse type input concentration at an intermediate location is defined as

$$
\left.c(x, y, t)=\begin{array}{rr}
c_{0}[1+\exp (-q t)] ; & 0<t \leq t_{0} \\
0 ; & t>t_{0}
\end{array}\right\} \quad \text { at } \quad x=x_{0}, \quad y=y_{0},
$$


where $t=t_{0}$ is the time when the source of the input concentration is removed forever, and $q\left[T^{-1}\right]$ is a decay parameter. The second boundary condition at the two extreme ends of horizontal medium is considered of homogeneous flux type, as

$$
\frac{\partial c}{\partial x}=0 \quad \text { and } \quad \frac{\partial c}{\partial y}=0 ; \quad x \rightarrow \infty, \quad y \rightarrow \infty, \quad t \geq 0 .
$$

Using an integral transformation (Crank 1975)

$$
T^{*}=\int_{0}^{t} V(m t) d t
$$

the left hand side term of Eq. 6 becomes $\left(\partial c / \partial T^{*}\right) . T^{*}$ is a new time variable, as $m$ (of dimension being inverse of that of the time variable) will appear in the denominator of its expression. $V(m t)$ is so chosen that this new time variable becomes zero for $t=0$; otherwise, the nature of the initial condition in new time domain will alter. It may be verified for its expression in Eq. 4. Further coordinate transformations

$$
X=x-x_{0}, \quad Y=y-y_{0}
$$

convert the location $\left(x=x_{0}, y=y_{0}\right)$ of the input concentration to $(X=0$, $Y=0$ ). Also Eq. 6 may be obtained in the form

$$
\frac{\partial c}{\partial T^{*}}=D_{x_{0}} \frac{\partial^{2} c}{\partial X^{2}}+D_{y_{0}} \frac{\partial^{2} c}{\partial Y^{2}}-u_{0} \frac{\partial c}{\partial X}-v_{0} \frac{\partial c}{\partial Y} .
$$

Similarly, the conditions given by Eqs. 7-9 may be written in new independent variables $\left(X, Y, T^{*}\right)$. Further, using another coordinate transformation

$$
z=X+Y \sqrt{\frac{D_{y_{0}}}{D_{x_{0}}}} \quad \text { or } \quad X+Y \sqrt{\frac{v_{0}}{u_{0}}},
$$

the two dimensional Eq. 12 is reduced into a one-dimensional equation

$$
\frac{\partial c}{\partial T^{*}}=D \frac{\partial^{2} c}{\partial z^{2}}-U \frac{\partial c}{\partial z}
$$

where $D=D_{x_{0}}\left(1+\frac{D_{y_{0}}^{2}}{D_{x_{0}}^{2}}\right)$ and $U=\left(u_{0}+v_{0} \sqrt{\frac{v_{0}}{u_{0}}}\right)$. Equation 13 is similar to the one used by Carnahan and Remer (1984). The point $(X=0, Y=0)$ corre- 
sponds to $(z=0)$. The initial and boundary conditions, Eqs. 7-9, will assume the respective form as

$$
\begin{gathered}
c\left(z, T^{*}\right)=c_{i} ; \quad z \geq 0, T^{*}=0, \\
\left.c\left(z, T^{*}\right)=\begin{array}{rr}
c_{0}\left(2-q T^{*}\right) ; & 0<T^{*} \leq T_{0}^{*} \\
0 ; & T^{*}>T_{0}^{*}
\end{array}\right\} ; \quad z=0, \\
\frac{\partial c}{\partial z}=0 ; \quad z \rightarrow \infty, \quad T^{*} \geq 0 .
\end{gathered}
$$

As the coefficients of ADE in $\left(z, T^{*}\right)$ domain are constants so Laplace integral transformation technique may be used to get the analytical solution of the initial and boundary value problem defined by Eqs. 14-17 (van Genuchten and Alves 1982), as (see the Appendix)

$$
\begin{aligned}
c\left(z, T^{*}\right) & =c_{i}+\exp \left(\frac{U z}{2 D}-\frac{U^{2} T^{*}}{4 D}\right)\left[\left(2 c_{0}-c_{i}\right) F\left(z, T^{*}\right)-q c_{0} G\left(z, T^{*}\right)\right] ; \\
0<T^{*} \leq & T_{0}^{*} \\
c\left(z, T^{*}\right) & =c_{i}+\exp \left(\frac{U z}{2 D}-\frac{U^{2} T^{*}}{4 D}\right)\left[c_{0}\left(2+q T_{0}^{*}\right)\left\{F\left(z, T^{*}\right)-F\left(z, T^{*}-T_{0}^{*}\right)\right\}\right. \\
& \left.-c_{i} F\left(z, T^{*}\right)-q c_{0}\left\{G\left(z, T^{*}\right)-G\left(z, T^{*}-T_{0}^{*}\right)\right\}\right] ; \quad T>T_{0}^{*}
\end{aligned}
$$

where

$$
\begin{aligned}
& F\left(z, T^{*}\right)=\frac{1}{2} \exp \left(\frac{U^{2} T^{*}}{4 D}-\frac{U z}{2 D}\right) \operatorname{erfc}\left(\frac{z}{2 \sqrt{D T^{*}}}-\frac{U T^{*}}{2 \sqrt{D T^{*}}}\right) \\
& +\frac{1}{2} \exp \left(\frac{U^{2} T^{*}}{4 D}+\frac{U z}{2 D}\right) \operatorname{erfc}\left(\frac{z}{2 \sqrt{D T^{*}}}+\frac{U T^{*}}{2 \sqrt{D T^{*}}}\right), \\
& G\left(z, T^{*}\right)=\frac{1}{2 U}\left(U T^{*}-z\right) \exp \left(\frac{U^{2} T^{*}}{4 D}-\frac{U z}{2 D}\right) \operatorname{erfc}\left(\frac{z}{2 \sqrt{D T^{*}}}-\frac{U T^{*}}{2 \sqrt{D T^{*}}}\right) \\
& +\frac{1}{2 U}\left(U T^{*}+z\right) \exp \left(\frac{U^{2} T^{*}}{4 D}+\frac{U z}{2 D}\right) \operatorname{erfc}\left(\frac{z}{2 \sqrt{D T^{*}}}+\frac{U T^{*}}{2 \sqrt{D T^{*}}}\right) .
\end{aligned}
$$

One can express this solution in terms of the original independent variables $(x, y, t)$ by using the appropriate transformation equations introduced at the different stages. By using Eqs. 4 and 10, the new time variable will have the expression 


$$
T^{*}=\frac{1}{m}[m t-(1-\cos m t)] .
$$

\section{RESULTS AND DISCUSSION}

To illustrate the analytical solutions (Eq. 18a, b), the values of different parameters are chosen from the literature cited in the Introduction as: initial pollution level parameter $c_{i}=0.01$, reference concentration and the decay parameter, both in the input condition in Eq. 8, are chosen as $c_{0}=1.0$, $q=0.0001(\text { day })^{-1}$, respectively, the velocity components $u_{0}=0.09 \mathrm{~km} / \mathrm{day}$, $v_{0}=0.009 \mathrm{~km} /$ day, and the dispersion coefficient components $D_{x_{0}}=0.1 \mathrm{~km}^{2} /$ day, $D_{y_{0}}=0.01 \mathrm{~km}^{2} /$ day. The concentration values are evaluated in the domain $0 \leq z, y[\mathrm{~km}] \leq 100$. The location of the point source (input concentration) is assumed at $x_{0}=30 \mathrm{~km}, y_{0}=30 \mathrm{~km}$. Figure 1a depicts the concentration values obtained from the solution in Eq. $18 \mathrm{a}$ in the presence of the source, i.e., in the time domain $t<t_{0}$ for $m t=8,11,14$. The

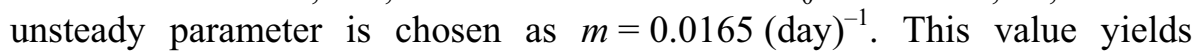
$t=484,666$, and 848 days . From Eq. 4 it may be observed that the velocity is alternatively minimum and maximum at these values of time; hence, they may be regarded as the time periods during the months of June of the second year, December of the same year and again June of the next year, from the introduction of the input concentration at $t=0$. The source of the input concentration is assumed to be eliminated at $t_{0}=909$ days, which corresponds to $m t=15$. Beyond this time, the concentration values evaluated from solution in Eq. $18 \mathrm{~b}$ are depicted in Fig. 1b, at $t=1030$, 1212, and 1394 days, which corresponds to $m t=17,20,23$, respectively, and represents the months of December of the third year, June and December of the next year, respectively. This scenario of velocity distribution either in aquifers or in rivers occurs in the tropical regions like India. Figure 1a shows that concentration values increase with position and time on either side of the point source (input concentration location), though the increase is much less in the left domain, that is, towards the origin $(x=0, y=0)$ in comparison of that at the same distances on the right domain, that is, away from the origin. Figure $1 \mathrm{~b}$ shows that, after the removal of the source of pollution, the concentration values at a particular position $(x, y)$ in the already polluted domain decrease with time. The domain nearer to the location of the source gets rehabilitated faster than the positions far away. To have more clarity on the solute mass distribution pattern originating from the point source, the concentration values in both time domains (before and after the elimination of the source) are also provided in Tables 1 and 2, respectively. It may be observed that according to the pulse type condition given in Eq. 8, the input concentration evaluated 
(a)

(b)
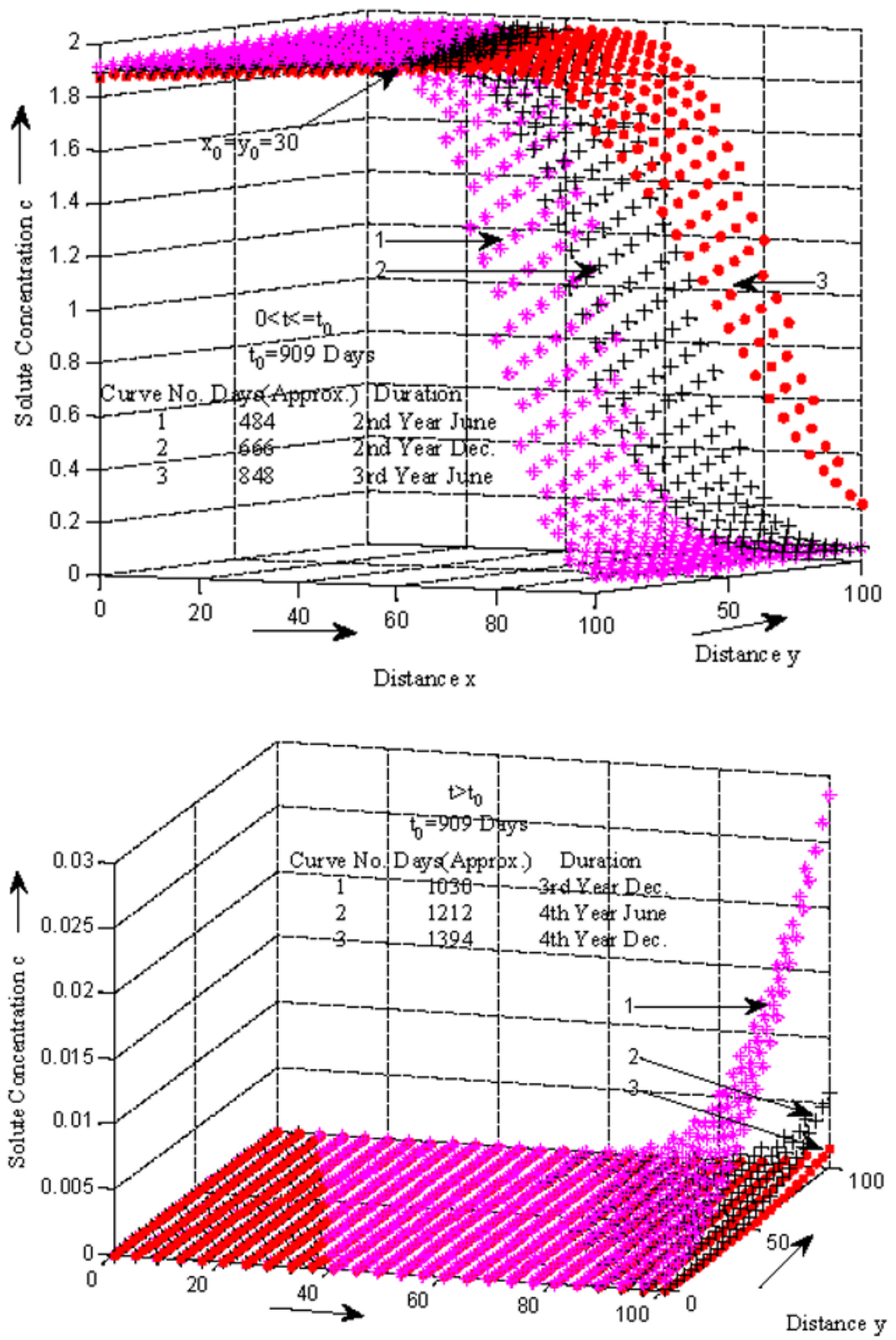

Distance $\mathrm{x}$

Fig.1. Solute mass horizontal dispersion pattern along and against the sinusoidally varying velocity: (a) in the presence of a point source, and (b) in the absence of a point source. 


\begin{tabular}{|c|c|c|c|}
\hline 1 & 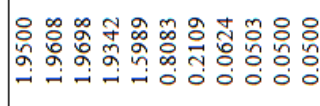 & 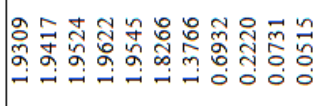 & 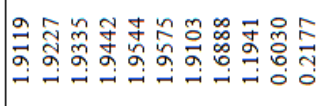 \\
\hline 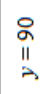 & 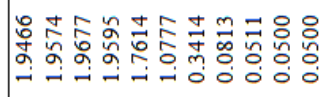 & 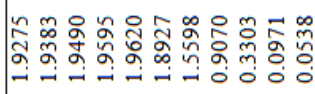 & 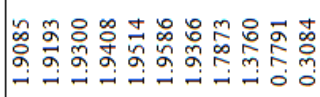 \\
\hline & 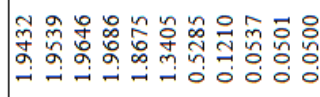 & 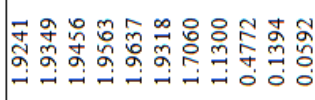 & 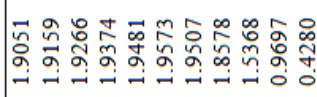 \\
\hline & 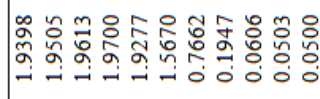 & 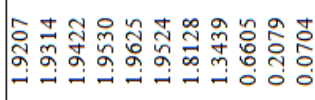 & 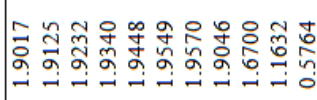 \\
\hline II & 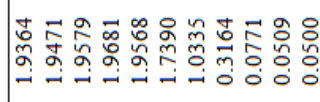 & 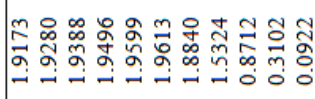 & 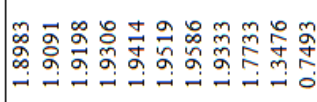 \\
\hline 4 & 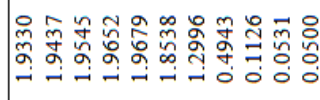 & 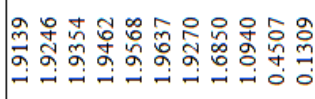 & 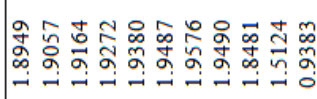 \\
\hline & 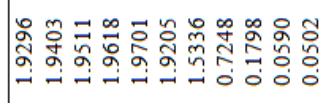 & 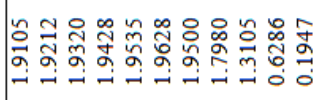 & 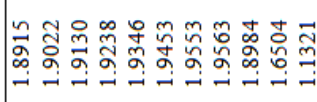 \\
\hline & 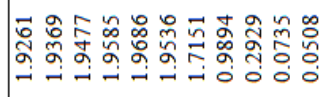 & 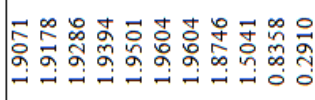 & $\begin{array}{l}\infty \\
\infty \\
\infty\end{array}$ \\
\hline 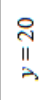 & 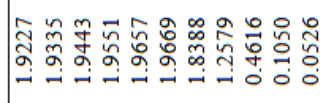 & 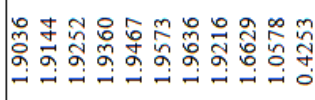 & 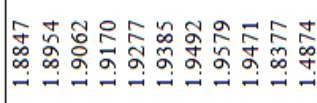 \\
\hline & 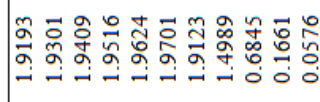 & 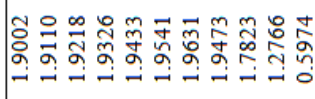 & 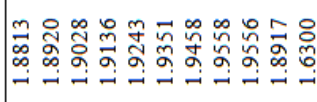 \\
\hline & 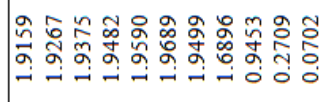 & 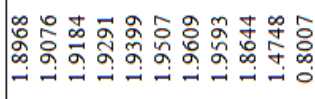 & 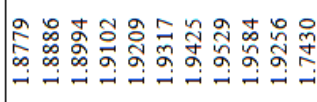 \\
\hline 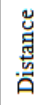 & 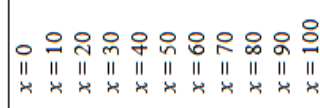 & 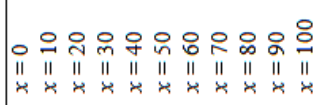 & 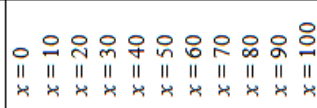 \\
\hline & $\underset{\stackrel{+}{+}}{+}$ & $:$ & $\stackrel{\infty}{\infty}$ \\
\hline
\end{tabular}




\begin{tabular}{|c|c|c|c|c|}
\hline \multirow{13}{*}{ | } & \\
\hline & \multicolumn{4}{|c|}{ 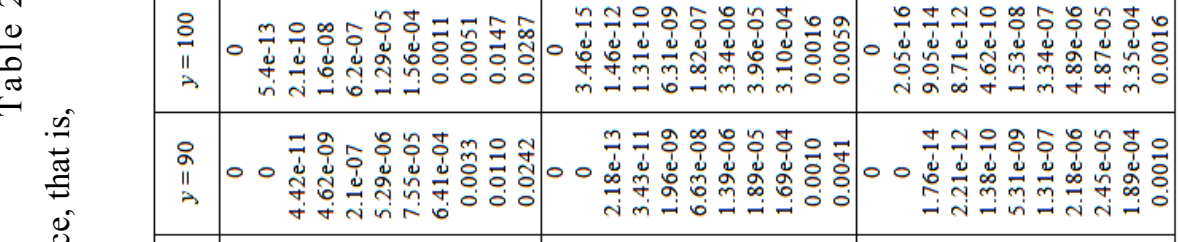 } \\
\hline & $\begin{array}{l}0 \\
11 \\
\text { I }\end{array}$ & 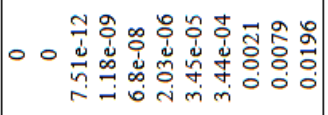 & 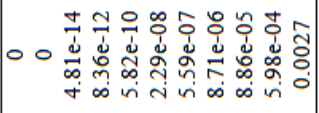 & 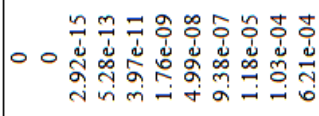 \\
\hline & $\stackrel{\circ}{i}$ & 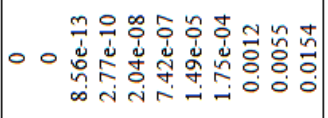 & 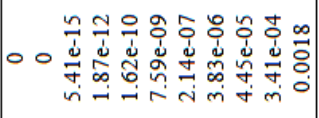 & 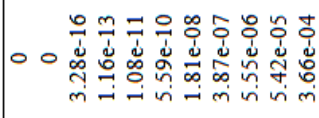 \\
\hline & \begin{tabular}{l}
8 \\
\hdashline \\
11 \\
2
\end{tabular} & 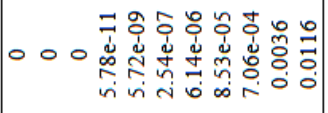 & 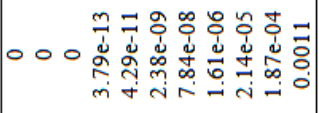 & 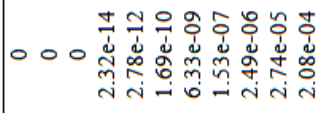 \\
\hline & $\begin{array}{l}\text { in } \\
\text { II } \\
\rightarrow\end{array}$ & 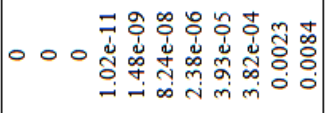 & 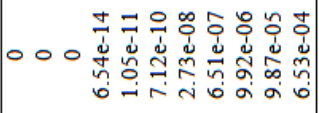 & 0 $00 \frac{\frac{n}{2}}{\frac{a}{n}}$ \\
\hline & $\begin{array}{l}\text { 암 } \\
\text { II } \\
\text { a }\end{array}$ & 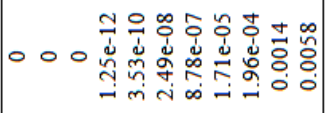 & 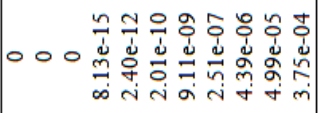 & 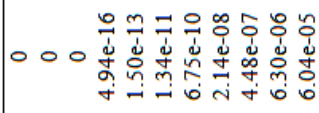 \\
\hline & 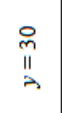 & 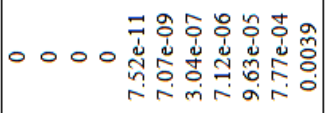 & ○ $000 \frac{m}{d}$ & 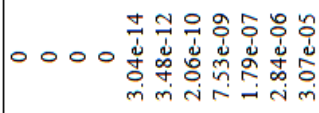 \\
\hline & $\underset{N}{\stackrel{ }{N}}$ & 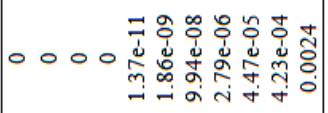 & 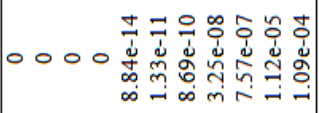 & 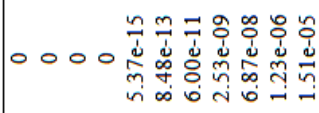 \\
\hline & $\stackrel{ }{\stackrel{\circ}{11}}$ & 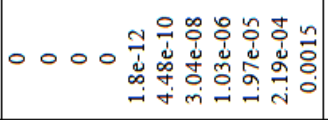 & 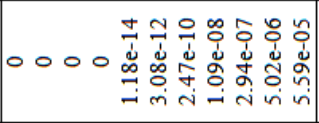 & ○ $000 \frac{0}{\frac{1}{2}}$ \\
\hline & $\begin{array}{l}0 \\
\| \\
\rightarrow\end{array}$ & 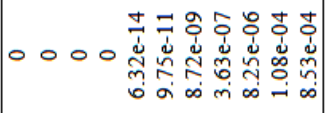 & 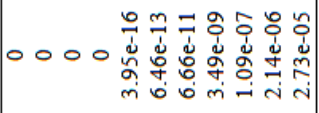 & 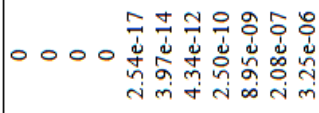 \\
\hline & 芯 & 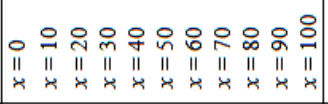 & 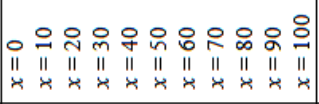 & 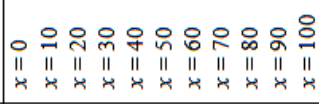 \\
\hline & $+\underset{\pi}{\infty}$ & ติ & & $\stackrel{m}{m}$ \\
\hline
\end{tabular}


from the solutions in Eq. 18a, b decreases very slowly with time in the former time domain and is zero in the latter time domain.

The new time variable $T^{*}$ has been computed from Eq. 19 for the values of old time variable $t$ considered above, and unsteady parameter $m=0.0165(\text { day })^{-1}$. We have $T^{*}=415,606,796,802,953,1176,1301$ days, for $t=484,666,848,909,1030,1212$, and 1394, respectively. It is evident that a value of new time variable is much less than the respective value of the old time variable. It means the concentration level at a position $(x, y)$ is obtained much earlier in case of sinusoidal flow domain than in case of uniform flow domain. The value of $m$ decides upon the periodicity, as in the present example it is almost half of a year. Similarly, for $m=0.01$ (day) $)^{-1}$, at $m t=0.05$, and 1.5, the non-dimensional value of the velocity from Eq. 4 will be 0.95 and 0.002 , respectively. In other words, the periodicity in this case occurs at the interval of approximately 15 days. The new time variable obtained from Eq. 19 will again be less than the respective old time variable in this set too. In this way Eq. 18a, b may be used for sinusoidal form of velocity of different periodicity.

In real cases, the current through the medium is seldom unidirectional; hence, the solute mass disperses both longitudinally as well as laterally, though that along the lateral direction may be much less than that in the longitudinal direction. In this regard, the values of the lateral components of velocity as well as of dispersion coefficient are considered much less (onetenth) of the respective longitudinal values but it is evident from the results that the concentration values along the lateral direction are significant. These results are compared with those of a one-dimensional model (Kumar and Kumar 1998) with the same assumptions. The concentration values on the same distance from the origin along the longitudinal direction have been found less in the two-dimensional model than those in the one-dimensional model. The solution given in Eq. 18a, b may also be used for other temporal dependent expressions of $V(m t)$, for example, for exponentially increasing $\exp (m t)$ or decreasing function $\exp (-m t)$. Choosing the value of $m$ appropriately, the pattern of time-dependence may be varied according to the situation and need. The results delivered from the analytical solution (Eq. 18a, b) are validated by the results obtained by solving the same initial and boundary value problem by a two level explicit method under the stability criterion. A very good agreement between the analytical and numerical results has been found.

The present dispersion problem has much deviation from idealistic conditions and incorporates some features nearer to real situations. For example, in the real world nowadays the dispersion of pollutants along different media of air, water, and soil has become a matter of great concern and there is a need to study such a problem through the solutions of advection diffusion 
equation. In a real situation, getting numerical solution is an easier way and cost effective but its validation by comparing it with an analytical solution of a similar problem is equally important. Therefore, it demands more and more analytical solutions of real problems and the present work is an effort in that direction. Analytical solutions have another advantage of providing better insight into the factors included in the problem, making it more realistic than any other solution. Most of the analytical solutions are approximate solutions, of which the Laplace transformation technique, as used in the present work, is the most viable method. In comparison to numerical models, analytical models and their solution provide more physical insight into conceptual mathematical behavior of the system. The obtained analytical solution is a benchmark to develop numerical codes and solutions. For practical application in subsurface hydrology, the local dispersion coefficients alone (eventually without molecular diffusion) may be used to a zeroth-order approximation of transport in mildly heterogeneous aquifers, consisting of a diffusion in the mean velocity field (Suciu 2010). A perfectly homogeneous aquifer is a highly idealized approximation of little practical relevance. The fitted diffusion coefficient larger than the local dispersion coefficient is only due to the scale effects induced by the spatial variability of the velocity field (Suciu 2014).

\section{CONCLUSIONS}

The main motive of the present work is to assess the concentration level in the vicinity as well as at far distances on both sides of the point source of a pollution mass, from one analytical solution. Another one was to use temporal dependence of dispersion coefficients in a two dimensional ADE while obtaining its analytical solution through Laplace Integral Transformation Technique. Such dependence may take care of the heterogeneity of the medium too, in addition to representing the unsteadiness behavior of the diffusing pollutant. It is always better to assess the pollution level more realistically by a two dimensional model than a one dimensional model. The solution obtained is based on the dispersion theory (Scheidegger 1957) valid in the water medium on the surface and in the aquifer.

Acknowledgment. The authors are thankful to the editors and reviewers for their constructive comments and valuable suggestions, which have helped improve the quality of the paper. 


\section{Appendix}

Using the following transformation

$$
c\left(z, T^{*}\right)=K\left(z, T^{*}\right) \exp \left(\frac{U z}{2 D}-\frac{U^{2} T^{*}}{4 D}\right),
$$

in Eq. 14 and the conditions given in Eqs. 15-17, we get a diffusive problem in new dependent variable as

$$
\begin{gathered}
\frac{\partial K}{\partial T^{*}}=D \frac{\partial^{2} K}{\partial z^{2}}, \\
K\left(z, T^{*}\right)=c_{i} \exp \left(-\frac{U z}{2 D}\right) ; \quad z \geq 0, \quad T^{*}=0, \\
\left.K\left(z, T^{*}\right)=\begin{array}{c}
c_{0}\left(2-q T^{*}\right) \exp \left(\frac{U^{2} T^{*}}{4 D}\right) ; \quad 0<T^{*} \leq T_{0}^{*} \\
0 ; \quad T^{*}>T_{0}^{*}
\end{array}\right\} ; \quad z=0, \\
\frac{\partial K}{\partial z}+\frac{U K}{2 D}=0 ; \quad z \rightarrow \infty, \quad T^{*} \geq 0 .
\end{gathered}
$$

Applying Laplace integral transformation in Eqs. A2-A5, we may get the solution $\bar{K}(z, p)$ as follows:

$$
\begin{aligned}
\bar{K}(z, p) & =c_{0}\left[2 \bar{K}_{1}(z, p)+q T_{0}^{*} \bar{K}_{2}(z, p)-q \bar{K}_{3}(z, p)\right] \\
& -c_{i} \bar{K}_{4}(z, p)+\frac{c_{i}}{\left(p-\frac{U^{2}}{4 D}\right)} \exp \left(-\frac{U z}{2 D}\right),
\end{aligned}
$$

where

$$
\begin{gathered}
\bar{K}_{1}(z, p)=\frac{1}{\left(p-\frac{U^{2}}{4 D}\right)}\left[1-\exp \left\{-\left(p-\frac{U^{2}}{4 D}\right) T_{0}^{*}\right\}\right] \exp \left(-\sqrt{\frac{p}{D}} z\right), \\
\bar{K}_{2}(z, p)=\frac{1}{\left(p-\frac{U^{2}}{4 D}\right)} \exp \left\{-\left(p-\frac{U^{2}}{4 D}\right) T_{0}^{*}\right\} \exp \left(-\sqrt{\frac{p}{D}} z\right)
\end{gathered}
$$




$$
\bar{K}_{3}(z, p)=\frac{1}{\left(p-\frac{U^{2}}{4 D}\right)^{2}}\left[1-\exp \left\{-\left(p-\frac{U^{2}}{4 D}\right) T_{0}^{*}\right\}\right] \exp \left(-\sqrt{\frac{p}{D}} z\right)
$$

and

$$
\bar{K}_{4}(z, p)=\frac{1}{\left(p-\frac{U^{2}}{4 D}\right)} \exp \left(-\sqrt{\frac{p}{D}} z\right)
$$

Now taking the inverse Laplace transform of Eq. A6, the solution $K\left(z, T^{*}\right)$ can be obtained as follows:

$$
\begin{aligned}
K\left(z, T^{*}\right)= & c_{0}\left[2 K_{1}\left(z, T^{*}\right)+q T_{0}^{*} K_{2}\left(z, T^{*}\right)-q K_{3}\left(z, T^{*}\right)\right] \\
& -c_{i} K_{4}\left(z, T^{*}\right)+\frac{c_{i}}{\left(p-\frac{U^{2}}{4 D}\right)} \exp \left(-\frac{U z}{2 D}\right),
\end{aligned}
$$

where

$$
\begin{aligned}
& K_{1}\left(z, T^{*}\right)=\left\{\begin{array}{rc}
F\left(z, T^{*}\right) ; & 0<T^{*} \leq T_{0}^{*} \\
F\left(z, T^{*}\right)-F\left(z, T^{*}-T_{0}^{*}\right) ; & T^{*}>T_{0}^{*}
\end{array},\right. \\
& K_{2}\left(z, T^{*}\right)=\left\{\begin{array}{rc}
0 ; & 0<T^{*} \leq T_{0}^{*} \\
F\left(z, T^{*}\right)-F\left(z, T^{*}-T_{0}^{*}\right) ; & T^{*}>T_{0}^{*}
\end{array},\right. \\
& K_{3}\left(z, T^{*}\right)=\left\{\begin{array}{rc}
G\left(z, T^{*}\right) ; & 0<T^{*} \leq T_{0}^{*} \\
G\left(z, T^{*}\right)-G\left(z, T^{*}-T_{0}^{*}\right) ; & T^{*}>T_{0}^{*}
\end{array}\right.
\end{aligned}
$$

and

$$
K_{4}\left(z, T^{*}\right)=F\left(z, T^{*}\right) .
$$

Applying the transformation A1 on this solution, the desired solution given in Eq. 18 may be obtained.

References

Aral, M.M., and B. Liao (1996), Analytical solutions for two-dimensional transport equation with time-dependent dispersion coefficients, J. Hydrol. Eng. 1, 1, 20-32, DOI: 10.1061/(ASCE)1084-0699(1996)1:1(20). 
Banks, R.B., and S.J. Jerasate (1962), Dispersion in unsteady porous media flow, J. Hydraul. Div. 88, 1-21.

Batu, V. (1989), A generalized two-dimensional analytical solution for hydrodynamic dispersion in bounded media with the first-type boundary condition at the source, Water Resour. Res. 25, 6, 1125-1132, DOI: 10.1029/ WR025i006p01125.

Batu, V. (1993), A generalized two-dimensional analytical solute transport model in bounded media for flux-type finite multiple sources, Water Resour. Res. 29, 8, 2881-2892, DOI: 10.1029/93WR00977.

Bear, J. (1972), Dynamics of Fluids in Porous Media, Elsevier, New York.

Carnahan, C.L., and J.S. Remer (1984), Non-equilibrium and equilibrium sorption with a linear sorption isotherm during mass transport through an infinite porous media: Some analytical solutions, J. Hydrol. 73, 3-4, 227-258, DOI: 10.1016/0022-1694(84)90002-7.

Chen, J.-S., and C.-W. Liu (2011), Generalized analytical solution for advectiondispersion equation in finite spatial domain with arbitrary time-dependent inlet boundary condition, Hydrol. Earth Syst. Sci. 15, 8, 2471-2479, DOI: 10.5194/hess-15-2471-2011.

Chen, J.-S., K.-H. Lai, C.-W. Liu, and C.-F. Ni (2012a), A novel method for analytically solving multi-species advective-dispersive transport equations sequentially coupled with first-order decay reactions, J. Hydrol. 420-421, 191-204, DOI: 10.1016/j.jhydrol.2011.12.001.

Chen, J.-S., C.-W. Liu, C.-P. Liang, and K.-H Lai (2012b), Generalized analytical solutions to sequentially coupled multi-species advective-dispersive transport equations in a finite domain subject to an arbitrary time-dependent source boundary condition, J. Hydrol. 456-457, 101-109, DOI: 10.1016/ j.jhydrol.2012.06.017.

Crank, J. (1975), The Mathematics of Diffusion, Oxford Univ. Press, Oxford.

Dagan, G. (1984), Solute transport in heterogeneous porous formations, J. Fluid Mech. 145, 151-177, DOI: 10.1017/S0022112084002858.

Domenico, P.A., and F.W. Schwartz (1997), Physical and Chemical Hydrogeology, John Wiley \& Sons, New York.

Ebach, E.A., and R.R. White (1958), Mixing of fluids flowing through beds of packed solids, AIChE J. 4, 2, 161-169, DOI: 10.1002/aic.690040209.

Freeze, R.A., and J.A. Cherry (1979), Groundwater, Prentice-Hall, New Jersey.

Fried, J.J. (1975), Groundwater Pollution, Developments in Water Science, Vol. 4, Elsevier, Amsterdam.

Fried, J.J., and M.A. Combarnous (1971), Dispersion in porous media. In: V.T. Chow (ed.), Advances in Hydroscience, Vol. 7, 169-282, Academic Press, New York.

Goltz, M.N., and P.V. Roberts (1986), Three-dimensional solutions for solute transport in an infinite medium with mobile and immobile zones, Water Resour. Res. 22, 7, 1139-1148, DOI: 10.1029/WR022i007p01139. 
Guerrero, J.S.P., L.C.G. Pimentel, T.H. Skaggs, and M.Th. van Genuchten (2009), Analytical solution of the advection-diffusion transport equation using a change-of-variable and integral transform technique, Int. J. Heat. Mass. Transfer 52, 13-14, 3297-3304, DOI: 10.1016/j.ijheatmasstransfer. 2009.02.002.

Jaiswal, D.K., A. Kumar, N. Kumar, and M.K. Singh (2011), Solute transport along temporally and spatially dependent flows through horizontal semi-infinite media: Dispersion proportional to square of velocity, J. Hydrol. Eng. 16, 3, 228-238, DOI: 10.1061/(ASCE)HE.1943-5584.0000312.

Javandel, I., C. Doughty, and C.F. Tasang (1984), Groundwater Transport: Handbook of Mathematical Models, Water Resources Monogr., Vol. 10, AGU, Washington DC.

Kumar, A., D.K. Jaiswal, and R.R. Yadav (2011), One-dimensional solute transport for uniform and varying pulse type input point source with temporally dependent coefficients in longitudinal semi-infinite homogeneous porous domain, Int. J. Math. Sci. Comput. 1, 2, 56-66.

Kumar, N., and M. Kumar (1998), Solute dispersion along unsteady groundwater flow in a semi-infinite aquifer, Hydrol. Earth Syst. Sci. 2, 1, 93-100, DOI: 10.5194/hess-2-93-1998.

Leij, F.J., T.H. Skaggs, and M.Th. van Genuchten (1991), Analytical solutions for solute transport in three-dimensional semi-infinite porous media, Water Resour. Res. 27, 10, 2719-2733, DOI: 10.1029/91WR01912.

Marshal, T.J., J.W. Holmes, and C.W. Rose (1996), Soil Physics, 3rd ed., Cambridge University Press, Cambridge.

Matheron, G., and G. De Marsily (1980), Is transport in porous media always diffusive? A counterexample, Water Resour. Res. 16, 5, 901-917, DOI: 10.1029/ WR016i005p00901.

Pickens, J.F., and G.E. Grisak (1981), Scale-dependent dispersion in a stratified gramular aquifer, Water Resour. Res. 17, 4, 1191-1211, DOI: 10.1029/ WR017i004p01191.

Sander, G.C., and R.D. Braddock (2005), Analytical solutions to the transient, unsaturated transport of water and contaminants through horizontal porous media, Adv. Water Resour. 28, 10, 1102-1111, DOI: 10.1016/j.advwatres. 2004.10.010.

Scheidegger, A. (1957), The Physics of Flow Through Porous Media, Univ. of Toronto Press, Toronto.

Serrano, S.E. (1995), Forecasting scale-dependent dispersion from spills in heterogeneous aquifers, J. Hydrol. 169, 1-4, 151-169, DOI: 10.1016/0022-1694 (94)02663-V.

Singh, M.K., N.K. Mahato, and P. Singh (2008), Longitudinal dispersion with timedependent source concentration in semi-infinite aquifer, J. Earth Syst. Sci. 117, 6, 945-949, DOI: 10.1007/s12040-008-0079-x. 
Singh, M.K., P. Singh, and V.P. Singh (2010), Analytical solution for twodimensional solute transport in finite aquifer with time-dependent source concentration, J. Eng. Mech. 136, 10, 1309-1315, DOI: 10.1061/ (ASCE)EM.1943-7889.0000177.

Suciu, N. (2010), Spatially inhomogeneous transition probabilities as memory effects for diffusion in statistically homogeneous random velocity fields, Phys. Rev. E 81, 5, 056301, DOI: 10.1103/PhysRevE.81.056301.

Suciu, N. (2014), Diffusion in random velocity fields with applications to contaminant transport in groundwater, Adv. Water Resour. 69, 114-133, DOI: 10.1016/j.advwatres.2014.04.002.

Sudicky, E.A., H.-T. Hwang, W.A. Illman, Y.-S. Wu, J.B. Kool, and P. Huyakorn (2013), A semi-analytical solution for simulating contaminant transport subject to chain-decay reactions, J. Contam. Hydrol. 144, 1, 20-45, DOI: 10.1016/j.jconhyd.2012.10.001.

Suresh Kumar, G., M. Sekhar, and D. Misra (2008), Time-dependent dispersivity of linearly sorbing solutes in a single fracture with matrix diffusion, J. Hydrol. Eng. 13, 4, 250-257, DOI: 10.1061/(ASCE)1084-0699(2008)13:4(250).

Tartakovsky, D.M. (2000), An analytical solution for two-dimensional contaminant transport during groundwater extraction, J. Contam. Hydrol. 42, 2-4, 273283, DOI: 10.1016/S0169-7722(99)00086-8.

Taylor, G. (1953), Dispersion of soluble matter in solvent flowing slowly through a tube, Proc. R. Soc. London A 219, 1137, 186-203, DOI: 10.1098/rspa. 1953.0139.

van Genuchten, M.Th., and W.J. Alves (1982), Analytical solutions of the onedimensional convective-dispersion solute transport equation, Tech. Bull. No. 1661, US Department of Agriculture, Washington D.C.

Warrick, A.W., J.W. Biggar, and D.R. Nielsen (1971), Simultaneous solute and water transfer for an unsaturated soil, Water Resour. Res. 7, 5, 1216-1225, DOI: $10.1029 /$ WR007i005p01216.

Wilson, J.L., and P.J. Miller (1978), Two-dimensional plume in uniform groundwater flow, J. Hydraul. Div. 104, 4, 503-514.

Yates, S.R. (1988), Three-dimensional radial dispersion in a variable velocity flow field, Water Resour. Res. 24, 7, 1083-1090, DOI: 10.1029/WR024i $007 \mathrm{p} 01083$.

Zoppou, C., and J.H. Knight (1997), Analytical solutions for advection and advectiondiffusion equations with spatially variable coefficients, ASCE J. Hydraul. Eng. 123, 2, 144-148, DOI: 10.1061/(ASCE)0733-9429(1997) 123:2(144).

Zoua, S., J. Ma, and A.D. Koussis (1996), Analytical solutions to non-Fickian subsurface dispersion in uniform groundwater flow, J. Hydrol. 179, 1-4, 237258, DOI: 10.1016/0022-1694(95)02830-7.

Received 12 February 2014 Received in revised form 23 May 2014 